\title{
Perioperative Concerns and Management of Patients with Right Atrial Thrombus in Chronic Constrictive Pericarditis Undergoing Pericardiectomy: A Case Series
}

Krishna P Gourav ${ }^{1}$, Srinath Damodaran ${ }^{2}$, Imran Bhat ${ }^{3}$, Sunder Negi ${ }^{4}$, Maramreddy Revanth ${ }^{5}$, Vinay Upadhyay ${ }^{6}$, Indranil Biswas ${ }^{7}$

\begin{abstract}
Chronic constrictive pericarditis (CCP) is a condition in which thickened, noncompliant pericardium encases heart structures. Association of right atrial (RA) thrombus in CCP imparts some challenges during the perioperative period. Perioperative echocardiography plays a vital role in managing this group of patients. This case series highlights the importance of perioperative echocardiography in managing the patients who had CCP with RA thrombus.

Keywords: Cardiopulmonary bypass, Constrictive pericarditis, Echocardiography, Right atrium thrombus.

Journal of Perioperative Echocardiography (2019): 10.5005/jp-journals-10034-1094
\end{abstract}

\section{INTRODUCTION}

Chronic constrictive pericarditis (CCP) is one of the common pericardial diseases in which pericardium is thickened and noncompliant. ${ }^{1}$ Pericardiectomy is the accepted definitive management of CCP with surgical operative mortality ranging from $6 \%$ to $12 \%{ }^{2}$ Detection of intracardiac thrombi in CCP alters the perioperative management, which includes application of anticoagulant therapy, use of minimally invasive technique to remove right atrial (RA) clot before pericardiectomy, alteration of surgical and anesthetic techniques, and involvement of cardiopulmonary bypass (CPB), since patients with such thrombi are at risk for embolization and right heart failure. Perioperative echocardiography may play an important role in this setting. We, hereby, report a case series of six patients who had CCP with right atrium thrombus/spontaneous echo contrast (SEC). This case series highlights the perioperative challenges while encountering such cases and the role of perioperative echocardiography in managing these patients.

\section{Case Description}

\section{Case 1}

A 22-year-old male patient diagnosed to be a case of CCP was planned for pericardiectomy. Surprisingly, on-table transthoracic echocardiography (TTE) done as an institutional protocol showed a large irregular hyperechoic mass $(30 \times 20 \mathrm{~mm})$ in the RA. Hence, the surgical plan was changed to pericardiectomy with RA clot removal under CPB (Fig. $1 \mathrm{~A}$ and Video 1). The central venous catheter (CVC) was inserted into the right internal jugular vein (IJV) and was advanced under the guidance of TTE and fixed at $2 \mathrm{~cm}$ proximal to clot to avoid dislodgement of RA thrombus. Transesophageal echocardiography (TEE) examination confirmed the TTE finding (Figs $1 \mathrm{~B}$ and $\mathrm{C}$ and Videos 2 and 3). Under the continuous monitoring of TEE, the pericardium was meticulously removed around superior vena cava (SVC) and inferior vena cava (IVC) with minimal manipulation of heart to cannulate SVC and IVC.
${ }^{1-4,7}$ Department of Anesthesia and Intensive Care, Postgraduate Institute of Medical Education and Research, Chandigarh, India

${ }^{5,6}$ Department of Cardiothoracic and Vascular Surgery, Postgraduate Institute of Medical Education and Research, Chandigarh, India

Corresponding Author: Krishna P Gourav, Department of Anesthesia and Intensive Care, Postgraduate Institute of Medical Education and Research, Chandigarh, India, Phone: +91 8194952345, e-mail: kpgmbbs777@gmail.com

How to cite this article: Gourav KP, Damodaran S, Bhat I, et al. Perioperative Concerns and Management of Patients with Right Atrial Thrombus in Chronic Constrictive Pericarditis Undergoing Pericardiectomy: A Case Series. J Perioper Echocardiogr 2019;7(1): 6-11.

Source of support: Nil

Conflict of interest: None

Under CPB, RA thrombus was evacuated, and the remaining part of pericardiectomy was carried out after termination of CPB. He was shifted to a cardiac surgical intensive care unit (ICU) with stable hemodynamics. The trachea was extubated after 7 hours of mechanical ventilation and shifted to ward after 3 days of uneventful ICU stay.

\section{Case 2}

A 40-year-old male patient who was suffering from constrictive pericarditis came to our hospital for pericardiectomy. On-table TTE confirmed the preoperative diagnosis with moderate pericardial effusion (PE) (Fig. 2A). However, TEE performed after induction of anesthesia detected a friable thrombus in the RA near SVC (Fig. 2B and Video 4). Hence, the surgical plan was changed from simple pericardiectomy to pericardiectomy plus thrombus evacuation under CPB. Similar to the first case, after limited pericardiectomy near SVC and IVC, bicaval cannulation was performed. Real-time monitoring of thrombus was done with TEE while handling the heart for IVC and SVC cannulation. Under CPB, RA clot was removed, and 


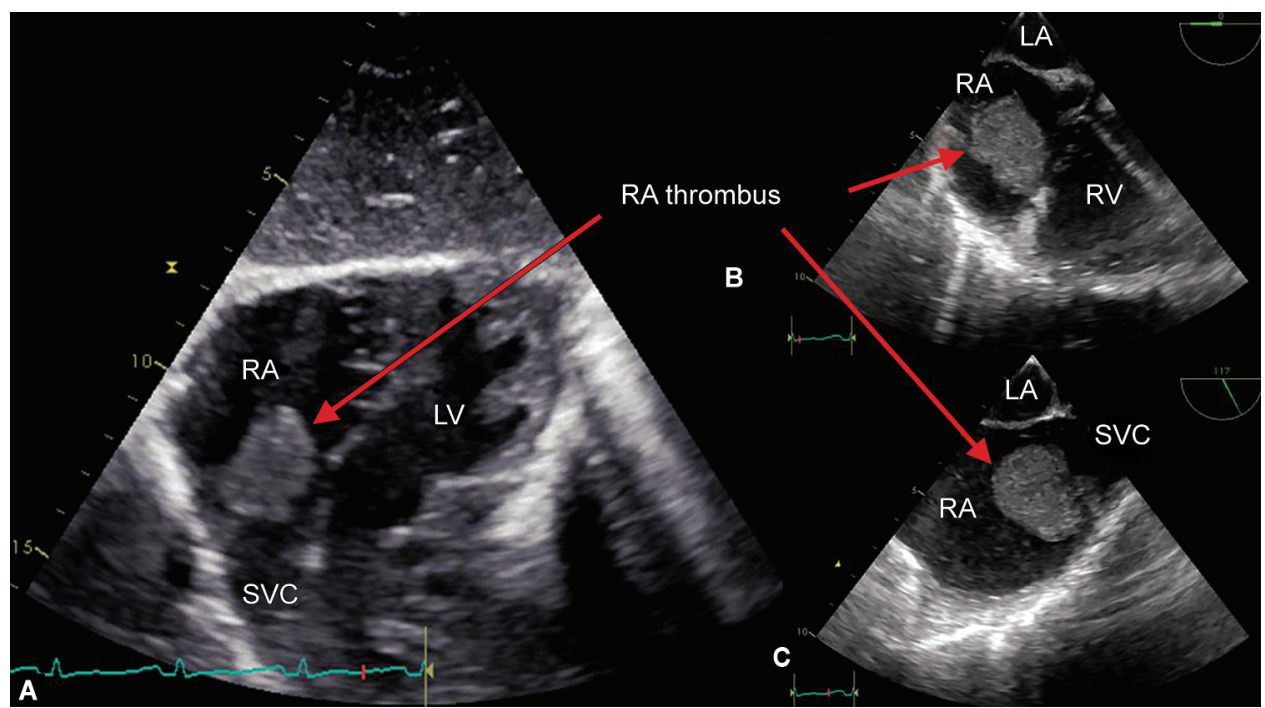

Figs $1 \mathrm{~A}$ to C: (A) Subcostal view in transthoracic echocardiography showing thrombus in the right atrium (RA); (B) Mid-esophageal four-chamber view in transesophageal echocardiography showing right atrial thrombus; (C) Mid-esophageal bicaval view in transesophageal echocardiography showing thrombus in the right atrium. LA, left atrium; LV, left ventricle; RV, right ventricle; SVC, superior vena cava
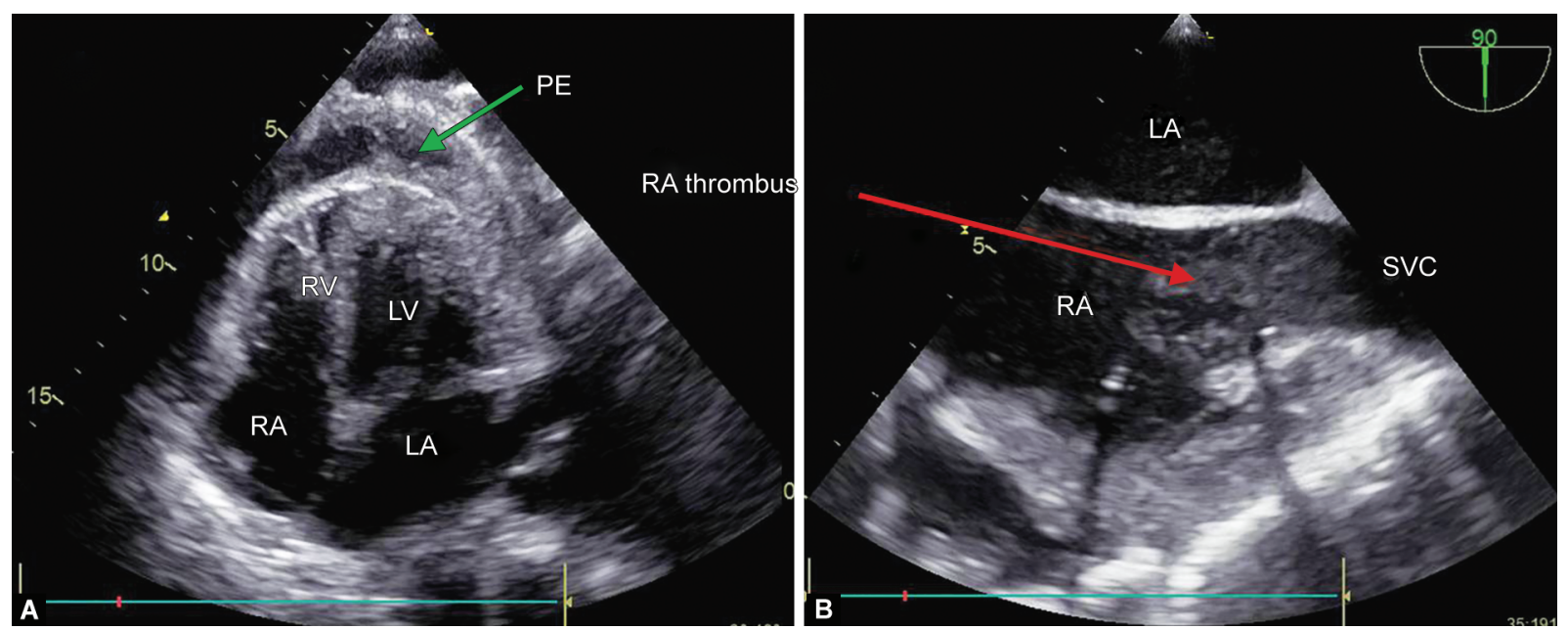

Figs 2A and B: (A) Apical four-chamber view in transthoracic echocardiography showing moderate pleural effusion; (B) Mid-esophageal bicaval view in transesophageal echocardiography showing friable thrombus in right atrium near superior vena cava. $L A$, left atrium; LV, left ventricle; RV, right ventricle

resection of the pericardium was performed after the termination of CPB.

\section{Case 3}

A 34-year-old male patient with the features of CCP posted for pericardiectomy. Transesophageal echocardiography performed after induction of anesthesia revealed a spontaneous echo contract in the RA which was not detected in on-table TTE (Fig. 3 and Video 5). However, no organized clot was detected in TEE, which was verified in multiple views. Hence, a standard pericardiectomy was performed via a median sternotomy without the use of CPB.

\section{Case 4}

A 44-year-old male patient, who was a known tubercular patient, admitted as a case of CCP and planned for pericardiectomy. Transesophageal echocardiography evaluation showed a $30 \mathrm{~mm}$ $\times 35 \mathrm{~mm}$ thrombus in RA near IVC in the mid-esophageal bicaval view (Fig. 4 and Video 6), which was not detected on on-table TTE.
The surgical plan was changed to clot removal and pericardiectomy. After careful pericardial dissection around SVC, SVC cannulation was carried out. Femoral venous cannulation was done instead of IVC and under the guidance of TEE, and the femoral venous cannula was advanced into the IVC, $3 \mathrm{~cm}$ proximal to the thrombus. Cardiopulmonary bypass was initiated, and thrombus was removed. Later on, pericardiectomy was carried out after termination of CPB.

\section{Case 5}

A 29-year-old male patient, who was a known tubercular patient, was admitted in the hospital with the features suggestive of constrictive pericarditis. Chest roentgenogram showed dullness in bilateral costophrenic angles. An ultrasound examination revealed bilateral massive pleural effusion (Fig. 5A). Transthoracic echocardiography showed pericardial thickening, dilated IVC, and more than $25 \%$ variation in mitral valve inflow, all suggestive of constrictive pericarditis (Fig. 5B). Hence, standard pericardiectomy was planned. A bilateral chest tube 


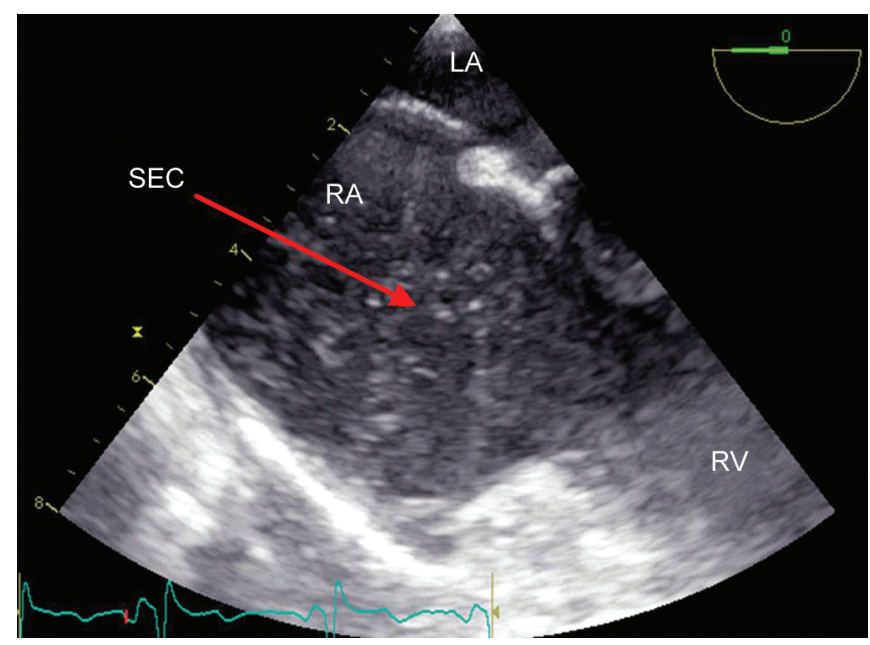

Fig. 3: Mid-esophageal four-chamber view in transesophageal echocardiography showing spontaneous echo contrast in the right atrium. LA, left atrium; RV, right ventricle

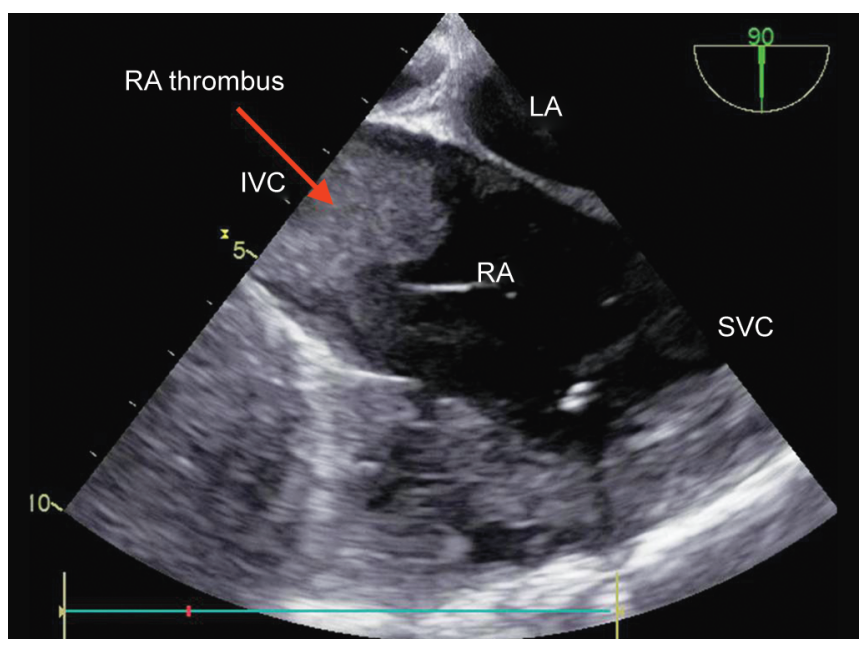

Fig.4: Mid-esophageal bicaval view in transesophageal echocardiography showing thrombus in the right atrium near inferior vena cava. LA, left atrium; SVC, superior vena cava

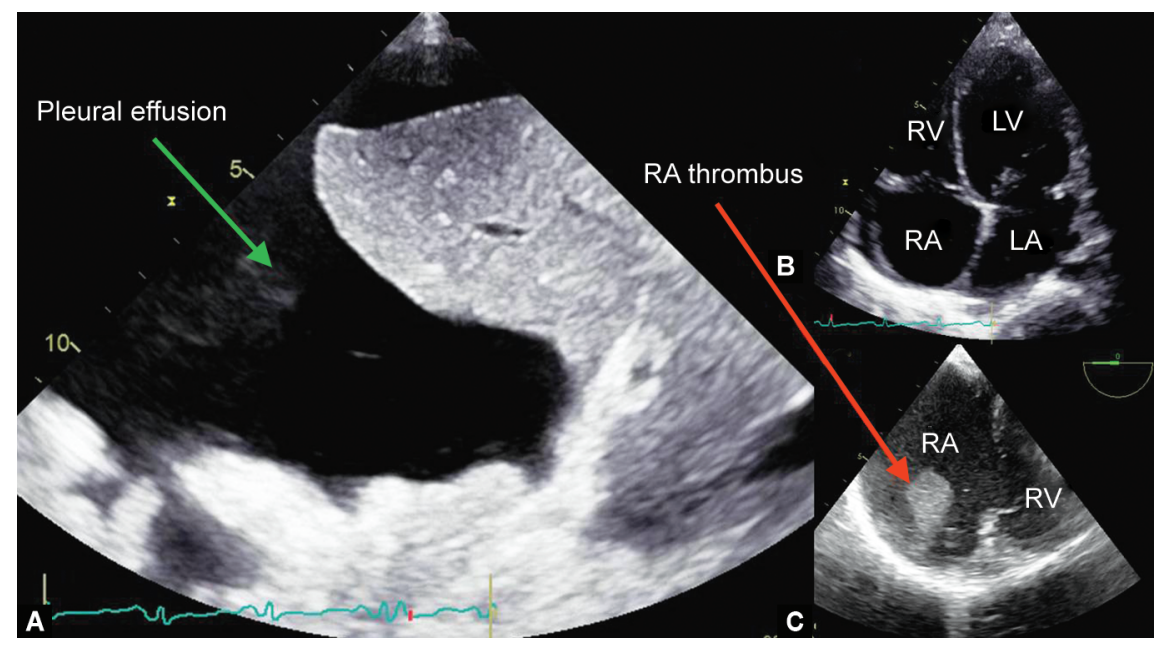

Figs 5A to C: (A) A transthoracic echocardiography image showing pleural effusion with collapsed right lung; (B) Apical four-chamber view in transthoracic echocardiography showing no right atrial clot; (C) Mid-esophageal four-chamber view in transesophageal echocardiography showing thrombus in the right atrium. LA, left atrium; LV, left ventricle; RV, right ventricle

insertion was placed to drain pleural effusion before induction of anesthesia as the patient was unable to lie supine on the operating table. TEE done after induction of anesthesia revealed a $40 \mathrm{~mm} \times 25 \mathrm{~mm}$ mass in the RA (Fig. 5C and Video 7). Hence, the surgical plan was changed. Similar to the first and second cases, CPB was initiated after bicaval cannulation, and RA thrombus evacuation was done followed by pericardiectomy after the termination of the bypass.

\section{Case 6}

A 37-year-old female patient weighing $47 \mathrm{~kg}$, diagnosed with a tubercular CCP came to our hospital for pericardiectomy. Preoperative TTE confirmed the diagnosis and detected a $20 \mathrm{~mm} \times$ $28 \mathrm{~mm}$ clot in the RA (Fig. 6A and Video 8). Low-molecular-weight heparin was started; however, even after 20 days of therapy, clot size in RA was not decreased. Hence, thrombolysis was attempted in ICU. Bedside TTE performed 3 days after thrombolysis showed complete resolution of RA clot (Fig. 6B and Video 9). One week later, elective pericardiectomy was done without the aid of CPB.

\section{Discussion}

Constrictive pericarditis is a pericardial disease in which a thickened noncompliant pericardium limits the filling of the ventricles during diastole. ${ }^{3-5}$ Various etiologies have been postulated for the development of CCP which includes viral pericarditis, collagen vascular disease, radiation, cardiac surgery, tuberculosis, etc. ${ }^{6}$ Patients with CCP usually presents with fatigue, pedal edema, and dyspnea. Abdominal discomfort, ascites, and pleural effusion are reported in later stages. The definitive diagnosis of CCP can be done by TTE, and it is considered as an initial imaging test of choice. ${ }^{7}$ The echocardiographic features of CCP are described in Table 1. Additional imaging tests like computer tomography (CT) and cardiac magnetic resonance (CMR) are required only in cases in whom echocardiographic findings are equivocal. Usually, CT imaging is done before redo operation to delineate cardiac, coronary, and pericardial anatomy. In addition to that, CMR is done in patients with short duration of CCP to assess the pericardial inflammation and to assess 

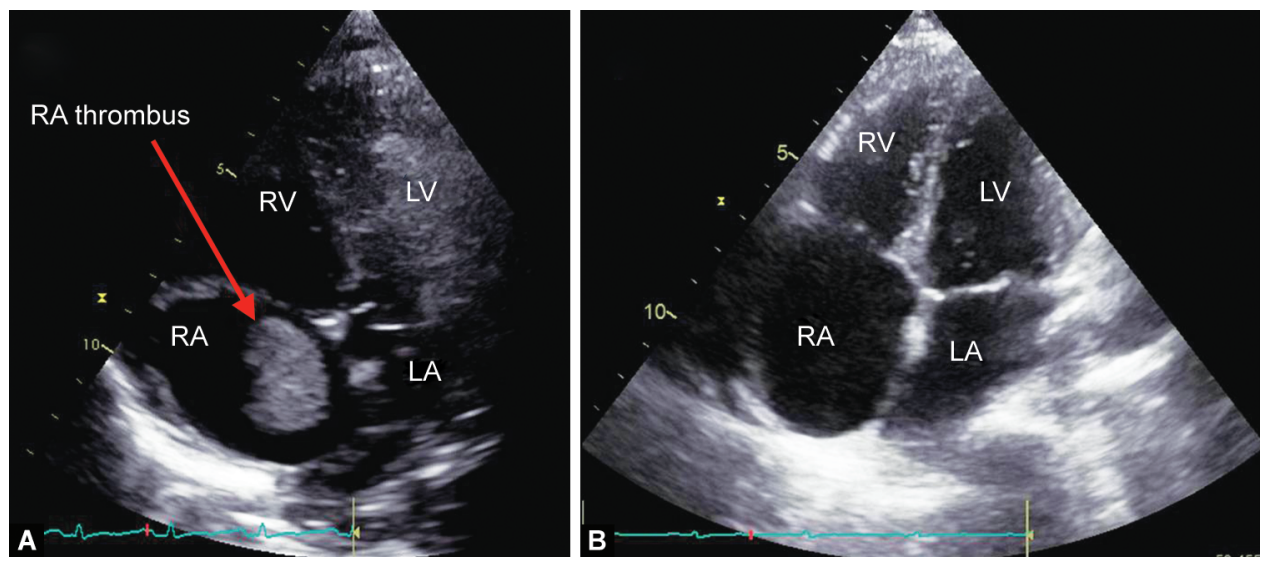

Figs $6 \mathrm{~A}$ and B: (A) An apical four-chamber view in transthoracic echocardiography showing thrombus in the right atrium; (B) Apical four-chamber view in transthoracic echocardiography showing complete resolution of thrombus after thrombolysis. LA, left atrium; LV, left ventricle; RV, right ventricle

Table 1: Features of chronic constrictive pericarditis in echocardiography

\begin{tabular}{ll}
\hline Variable & CCP features \\
\hline Septal motion & Respiratory shift \\
Mitral E/A ratio & $>1.5$ \\
Mitral deceleration time & $<160$ \\
(milliseconds) & \\
Mitral inflow respiratory & Mitral peak $E$ velocity $>25 \%$ \\
variation & usually present \\
Hepatic vein Doppler & Expiratory diastolic flow reversal \\
Mitral septal annular $e^{\prime}$ & Usually $>7$ cm/seconds \\
Mitral lateral annular $e^{\prime}$ & Less than septal $e^{\prime}$ \\
Pericardium & Thickening and calcification \\
Pleural effusion & May be visualized \\
\hline
\end{tabular}

recurrent constriction developed after cardiac surgery or after pericardiectomy. ${ }^{8,9}$

Cardiac thrombus is typically located in the atria, more often in the left side with smooth contoured, which moves synchronously with the adjacent heart wall during the cardiac cycle. ${ }^{10,11}$ Although left atrium thrombus is common, RA thrombus reported with a prevalence of $7 \%$ and is usually found in patients with the sluggish flow, endocardial damage, or foreign bodies in right-sided heart. ${ }^{12,13}$ It can cause tricuspid valve obstruction, pulmonary artery (PA) embolism, right heart failure, and systemic embolism. ${ }^{13-15}$ It has been reported in cases with dilated cardiomyopathy, idiopathic enlargement of RA, fibroelastosis, infective endocarditis, tricuspid valve replacement, right-sided pacemaker leads, indwelling CVC, restrictive cardiomyopathy due to amyloidosis, and in few cases with CCP. ${ }^{16,17}$

In CCP, RA thrombus was first reported in 1984 by Akiyama et al. ${ }^{18}$ The possible mechanism for the development of the RA thrombus in CCP includes stasis of blood in atrium due to impaired ventricular filling during diastole, inflammatory process, endocardial abnormalities, or atrial fibrillation. ${ }^{19}$ Apart from intracardiac thrombus in CCP, few case reports mentioned the presence of thrombus in the IJV, SVC, and apical region of the left ventricle. ${ }^{14,20}$ TTE and TEE play an important role in diagnosing cardiac mass. In developing countries, patients have been there in a long-waiting list before scheduling for cardiac surgery. During this waiting period, the patient may deteriorate further, which needs evaluation before anesthesia induction. In our institute, we made a routine protocol to perform TTE before induction of anesthesia to confirm the diagnosis. This practice not only helps in confirmation of diagnosis but also helps in identifying additional findings which will benefit in managing the patients. In case 1, on-table TTE helped in diagnosing thrombus in RA. Sometimes, TTE failed to pick up a thrombus, which might be due to the obscured image of RA by the thickened calcified pericardium. Hence, TEE is considered superior to TTE in assessing cardiac pathology because of its proximity to the structures without the interposition of pulmonary or parietal structures and due to the use of high-resolution transducers. In our index cases 2, 4, and 5, the TTE failed to detect RA thrombus. Furthermore, TEE is superior for the demonstration of the interatrial septum, and thus, an extension of any thrombi through patent foramen ovale can be better appreciated. ${ }^{21}$ The RA thrombus can be confused with congenital structures such as persistent eustachian or thebesian valves, Chiari network, atrial septal aneurysms, intracardiac vegetations, devices, and myxoma. Hence, a careful evaluation has to be done before confirming the RA thrombus to avoid unnecessary interventions. ${ }^{22-24}$

Patients having RA thrombus in CCP needs surgical thrombectomy apart from pericardiectomy, which requires $\mathrm{CPB}$. Since this is a high-risk procedure due to the possibility of embolization, thrombolysis may be advocated first before pericardiectomy as it is simple, widely applicable, and due to its rapid treatment. ${ }^{25}$ Also, the RA thrombi behave same as plateletrich red thrombi disappearing slowly with oral anticoagulants or more early with thrombolysis. Thrombolysis can be performed using streptokinase over 24 hours or recombinant tissue plasminogen activator over 2 hours. Post-thrombolysis evaluation can be carried out with bedside TTE. Then, the patient should be placed on anticoagulant therapy with unfractionated heparin till pericardiectomy. However, giant atrial thrombus may not resolve with thrombolysis. Surgical evacuations should be considered if thrombolysis failed or contraindicated. We attempted thrombolysis in index case 6 as it was detected in the preoperative period and due to its small size. However, in index cases 1, 2, 4, and 5, surgical thrombectomies were done instead of thrombolysis as the thrombus was identified in the operating room. In index case 1 , thrombus was diagnosed after induction of anesthesia with TTE, whereas in cases 2,4 , and 5, thrombi were diagnosed with the help of TEE after surgical incision. Monitoring with TTE or TEE plays an important role during thrombolysis or surgical evacuation to assess 
right ventricle (RV) function as clot could migrate to $P A$ at any point of time leading to RV failure.

Another novel technique in the armamentarium of thrombus removal is AngioVac. It is the recently developed technique in the removal of large thrombus, fresh thrombus from the vascular system. This AngioVac aspiration system consists of a 22-F catheter with a balloon-actuated tip, a circuit with a built-in filter, a centrifugal pump head, and a reinfusion cannula. ${ }^{26,27}$ It uses a perfusion circuit to forcefully aspirate clot or intravascular thrombus en bloc. Risk of RA/RV/IVC injuries and embolization of the thrombus are few rare complications associated with it. ${ }^{28}$ Currently, this technique was employed for the removal of acute ( $<3$ months) and soft clots, particularly useful in patients who cannot undergo thrombolytic therapy or surgery. Till date, no patient was treated with AngioVac technique in patients with RA thrombus with CCP.

The CVC is routinely placed during cardiac surgeries. Right IJV is preferred over other veins due to its straight course and less prone for lung injury. However, in patients with RA thrombus, right IJV cannulation can dislodge the thrombus. Hence, the right IJV cannulation should be done under caution. Before CVC cannulation, the right IJV should be scanned with ultrasound thrombus, and if found, alternative great veins should be considered. In addition to it, CVC should be advanced over guide wire under the guidance of TTE or TEE to prevent dislodgement of thrombus in the RA. In all of our cases, CVC was inserted into the IJV after scanning with ultrasound, and it was advanced under the guidance of TTE/TEE to prevent embolization of RA thrombus.

Usually, the pericardiectomy is carried out without implementing CPB. However, CPB may be necessary for it if intervention to coronary artery disease or valvular heart disease is required. ${ }^{29,30}$ The main concern in pericardiectomy is the manipulation of heart while resecting the pericardium. If CCP is associated with RA thrombus, embolization can occur while handling the heart. Hence, surgical thrombectomy should be considered before pericardiectomy to prevent embolization which requires CPB. To initiate CPB, bicaval cannulation is required. Sometimes, the RA thrombus can extend into SVC and IVC, hence, meticulous dissection to be done for cannulation. Sometimes, more distal cannulation of SVC and IVC may be required to avoid thrombus dislodgement. In our index case 2 , SVC cannulation was attempted superior to clot. For index case 4, we purposefully avoided IVC cannulation to prevent thrombus dislodgement. Real-time monitoring of RA thrombus was done in all our cases with the help of TEE while handling the heart to guide the surgeon. Sometimes, acute pulmonary embolism can occur while handling, and it can be associated with hemodynamic compromise, RV hypokinesia, congestive heart failure, or even death. Catecholamine and inodilator infusion should be prepared in advance and administered as soon as blood pressure falls below $90 \mathrm{~mm} \mathrm{Hg}$ or severe RV dysfunction is suspected. Transesophageal echocardiography also helps in assessing the volume status, severity of atrioventricular valve regurgitation, hemodynamic status, and ventricular function after pericardiectomy as it can decrease after pericardiectomy. Assessment of the main PA and branch PA should be done after RA clot removal as embolization can occur to PA.

\section{Conclusion}

This case series illustrates the importance of perioperative echocardiography's role in managing the cases with RA thrombus in CCP during the perioperative period, which can benefit patient survival.

\section{Author Contributions}

Krishna P Gourav, Srinath Damodaran, Sunder Negi, Maramreddy Revanth, Vinay Upadhyay and Indranil Biswas managed the case and prepared the manuscript. Imran Bhat prepared the manuscript.

\section{References}

1. Chen R, Lai CP. Clinical characteristics and treatment of constrictive pericarditis in Taiwan. Circ J 2005;69(4):458-460. DOI: 10.1253/ circj.69.458.

2. DeValeria PA, Baumgartner WA, Casale AS, et al. Current indications, risks, and outcome after pericardiectomy. Ann Thorac Surg 1991;52(2):219-224. DOI: 10.1016/0003-4975(91)91339-w.

3. Troughton RW, Asher CR, Klein AL. Pericarditis. Lancet 2004;363(9410):717-727. DOI: 10.1016/S0140-6736(04)15648-1.

4. Maisch B, Seferovic PM, Ristic AD, et al. Guidelines on the diagnosis and management of pericardial diseases executive summary; the task force on the diagnosis and management of pericardial diseases of the European Society of Cardiology. Eur Heart J 2004;25(7):587-610. DOI: 10.1016/j.ehj.2004.02.002.

5. Little WC, Freeman GL. Pericardial disease. Circulation 2006;113(12):1622-1632. DOI: 10.1161/CIRCULATIONAHA.105. 561514.

6. Bertog SC, Thambidorai SK, Parakh K, et al. Constrictive pericarditis: etiology and cause-specific survival after pericardiectomy. J Am Coll Cardiol 2004;43(8):1445-1452. DOI: 10.1016/j.jacc.2003.11.048.

7. Klein AL, Abbara S, Agler DA, et al. American society of echocardiography clinical recommendations for multimodality cardiovascular imaging of patients with pericardial disease: endorsed by the society for cardiovascular magnetic resonance and society of cardiovascular computed tomography. J Am Soc Echocardiogr 2013;26(9):965-1012.e15. DOI: 10.1016/j.echo.2013.06.023.

8. Bogaert J, Francone M. Pericardial disease: value of CT and MR imaging. Radiology 2013;267(2):340-356. DOI: 10.1148/ radiol.13121059.

9. Frank H, Globits S. Magnetic resonance imaging evaluation of myocardial and pericardial disease. J Magn Reson Imaging 1999;10(5):617-626. DOI: 10.1002/(sici)1522-2586(199911)10:53.0.co;2-z.

10. Burke A, Jeudy JJr, Virmani R. Cardiac tumours: an update-cardiac tumours. Heart 2008;94(1):117-123. DOI: 10.1136/hrt.2005.078576.

11. Restrepo CS, Largoza A, Lemos DF, et al. CT and MR imaging findings of benign cardiac tumors. Curr Probl Diagn Radiol 2005;34(1):12-21. DOI: 10.1067/j.cpradiol.2004.10.002.

12. Ogren $M$, Bergqvist $D$, Eriksson $H$, et al. Prevalence and risk of pulmonary embolism in patients with intracardiac thrombosis: a population-based study of 23796 consecutive autopsies. Eur Heart J 2005;26(11):1108-1114. DOI: 10.1093/eurheartj/ehi130.

13. London AR, Runge PJ, Balsam RF, et al. Large right atrial thrombi surrounding permanent transvenous pacemakers. Circulation 1969;40(5):661-664. DOI: 10.1161/01.cir.40.5.661.

14. Chakko S, Richards FIII. Right-sided cardiac thrombi and pulmonary embolism. Am J Cardiol 1987;59(1):195-196. DOI: 10.1016/S00029149(87)80114-5.

15. Pliam MB, McGough EC, Nixon W, et al. Right atrial ball-valve thrombus: a complication of central venous alimentation in an infant. J Thorac Cardiovasc Surg 1979;78(4):579-582. DOI: 10.1016/ S0022-5223(19)38087-0.

16. Yilmaz M, Gurlertop Y, Erdogan F. Right atrial thrombus following closure of an atrial septal defect. Heart 2003;89(7):726. DOI: 10.1136/ heart.89.7.726.

17. Burns KE, MCLaren A. Catheter-related right atrial thrombus and pulmonary embolism: a case report and systematic review of the literature. Can Respir J 2009;16(5):163-165. DOI: 10.1155/2009/ 751507.

18. Akiyama K, Nakae S, Imamura E, et al. A case of right atrial thrombus associated with constrictive pericarditis. Rinshou Kyoubu Geka 1984;4(5):607-612. 
19. Fowler N. Constrictive pericarditis: new aspects. Am J Cardiol 1982;50(5):1014-1017. DOI: 10.1016/0002-9149(82)90410-6.

20. Pahade A, Tewari P. Major vessel venous thrombosis in patients of post tubercular chronic constrictive pericarditis undergoing pericardiectomy: a rare scenario. Ann Card Anaesth 2017;20(3): 348-350. DOI: 10.4103/aca.ACA_77_16.

21. Manning WJ, Weintraub RM, Waksmonski CA, et al. Accuracy of transesophageal echocardiography for identifying left atrial thrombi: a prospective, intraoperative study. Ann Intern Med 1995;123(11):817-822. DOI: 10.7326/0003-4819-123-11-19951201000001.

22. Panidis IP, Kotler MN, Mintz GS, et al. Clinical and echocardiographic features of right atrial masses. Am Heart J 1984;107(4):745-758. DOI: 10.1016/0002-8703(84)90324-7.

23. Felner JM, Churchwell AL, Murphy DA. Right atrial thromboemboli: Clinical, echocardiographic and pathophysiologic manifestations. J Am Coll Cardiol 1984;5(5):1041-1051. DOI: 10.1016/s07351097(84)80069-8.

24. YarnalJR,SmileyWH. Right atrial mass simulated echocardiographically by a swan-Ganz catheter. Chest 1978;74(4):478-479. DOI: 10.1378/ chest.74.4.478-a.
25. Goldhaber SZ, Nagel JS, Theard M, et al. Treatment of right atrial thrombus with urokinase. Am Heart J 1988;115(4):894-897. DOI: 10.1016/0002-8703(88)90894-0.

26. Todoran TM, Sobieszczyk PS, Levy MS, et al. Percutaneous extraction of right atrial mass using the AngioVac aspiration system. J Vasc Interv Radiol 2011;22(9):1345-1347. DOI: 10.1016/j.jvir.2011.04.004.

27. Sakhuja R, Gandhi S, Rogers RK, et al. A novel endovenous approach for treatment of massive central venous or pulmonary arterial thrombus, mass, or vegetation: the AngioVac suction cannula and circuit [abstract]. J Am Coll Cardiol 2011;57(14):E1535. DOI: 10.1016/ S0735-1097(11)61535-0.

28. EIMallah W, Kalra N, Wiisanen M, et al. AngioVac catheter extractions: a novel technique for cardiac mass extraction. Learning curve and predictors of success [abstract]. Catheter Cardiovasc Interv 2014;83(Suppl S1):S117.

29. Kudaka M, Koja K, Kuniyoshi Y, et al. A case report of surgical treatment of constrictive pericarditis with coronary artery disease. Nihon Kyobu Geka Gakkai Zasshi 1997;45(11):1880-1883.

30. Zengbin F, Zhiwei L, Chaoman Z. Coronary heart disease complicated with chronic constrictive pericarditis: a case report. Chin J Clin Thorac Cardiovasc Surg 2006;13(3):63.

Video 1: Subcostal view in transthoracic echocardiography showing thrombus in right atrium

Video 2: Mid-esophageal 4-chamber view in transesophageal echocardiography showing right atrium thrombus

Video 3: Mid-esophageal bicaval view in transesophageal echocardiography showing thrombus in right atrium

Video 4: Mid-esophageal bicaval view in transesophageal echocardiography showing a friable thrombus in right atrium near superior vena cava

Video 5: Mid-esophageal 4-chamber view in transesophageal echocardiography showing spontaneous echo contrast in right atrium

Video 6: Mid-esophageal bicaval view in transesophageal echocardiography showing a friable thrombus in right atrium near inferior vena cava

Video 7: Mid-esophageal 4-chamber view in transesophageal echocardiography showing thrombus in right atrium

Video 8: An apical 4-chamber view in transthoracic echocardiography showing thrombus in right atrium

Video 9: An apical 4-chamber view in transthoracic echocardiography showing complete resolution of thrombus in right atrium after thrombolysis 